Journal of Computer Science 5 (10): 704-710, 2009

ISSN 1549-3636

(C) 2009 Science Publications

\title{
A New Approach of Adaptive Network-Based Fuzzy Inference System Modeling in Laser Processing-A Graphical User Interface (GUI) Based
}

\author{
${ }^{1}$ Sivarao, ${ }^{2}$ Peter Brevern and ${ }^{2}$ N.S.M. El-Tayeb \\ ${ }^{1}$ Faculty of Manufacturing Engineering, University Technical Malaysia Melaka, Malacca, Malaysia \\ ${ }^{2}$ Faculty of Engineering and Technology, Multimedia University, Malacca, Malaysia
}

\begin{abstract}
Problem statement: The power of Artificial Intelligent (AI) becomes more authoritative when the system is programmed to cater the need of complex applications. MATLAB 2007B, integrating artificial intelligent system and Graphical User Interface (GUI) has reduced researchers' fear-to-model factor due to unfamiliarity and phobia to produce program codes. Approach: In this study, how GUI was developed on Matlab to model laser machining process using Adaptive Networkbased Fuzzy Inference System (ANFIS) was presented. Laser cutting machine is widely known for having the most number of controllable parameters among the advanced machine tools, hence become more difficult to engineer the process into desired responses; surface roughness and kerf width. Mastering both laser processing and ANFIS programming are difficult task for most researchers, especially for the difficult to model processes. Therefore, a new approach was ventured, where GUI was developed using MATLAB integrating ANFIS variables to model the laser processing phenomenon, in which the numeric and graphical output can be easily printed to interpret the results. Results: To investigate ANFIS variables' characteristic and effect, error was analyzed via Root Mean Square Error (RMSE) and Average Percentage Error (APE). The RMSE values were then compared among various trained variables and settings to finalize best ANFIS predictive model. The results found was very promising and proved that, even a person with shallow knowledge in both artificial intelligence and laser processing can actually train the experimental data sets loaded into GUI, test and optimize ANFIS variables to make comparative analysis. Conclusion: The details of modeled work with prediction accuracy according to variable combinations were premeditated on another paper. The findings were expected to benefit precision machining industries in reducing their down time and cost as compared to the traditional way of trial and error method.
\end{abstract}

Key words: ANFIS modeling, laser machining, GUI development, surface finish, kerf width

\section{INTRODUCTION}

Laser cutting machine is one of new types in advanced machine tools that have been developed to improve machining process. However, when the machining process becomes more complex and difficult, the controllable parameters are proportionally increased to cater the need. This is where the computers replace human intuition when sensitive inputs need to be monitored and controlled by logical decision all the time. The result is the creation of artificial intelligence; fuzzy logic, neural networks and ANFIS to generate better decision over critical data. After years of development, MATLAB is now capable of doing programming job the same as C/C\#, Visual Basic and FORTRAN or better. It can also be integrated with the functionality of other programs which makes MATLAB far more versatile than their predecessor. Since MATLAB is advanced software that can compute complex algorithm, most of the developed GUIs are designed to be simple and user friendly. A MATLAB based Graphical User Interface (GUI) has been developed to design and optimize the LQG controllers which were then applied to antennas and radio telescopes ${ }^{[1]}$. The GUI was developed to simplify the design process of an LQG controller for those with limited control engineering background. The user is asked to manipulate the GUI sliders and radio buttons to watch the antenna performance. MATLAB's GUI tool was also benefited by researchers for artificial intelligent applications ${ }^{[2]}$, where system was proposed to discriminate between Amplitude Modulation (AM), Frequency Modulation (FM), Double Sideband (DSB), Upper Sideband (USB), Lower Sideband (LSB) and Continuous Wave (CW) modulations. A new fuzzy rule acquisition method was developed for tool wear estimation using radial basis networks to find the

Corresponding Author: Sivarao, Faculty of Manufacturing Engineering, University Technical Malaysia Melaka, 
optimal rules combination to compose fuzzy reasoning mechanism and options related to the Membership Functions $(\mathrm{MFs})^{[3]}$. On the other hand, CNC tool wear detection using Neurofuzzy classification system has been conducted, in which the investigation uses three different types of membership function for ANFIS training and compared their differences of accuracy rate of the turning tool-state detection ${ }^{[4]}$. A Fuzzy Neural Network classification application for drill tool-failure detection with fuzzy logic based decision mechanism was developed to determine tool wear condition using drilling forces ${ }^{[5]}$.

In this study, an ANFIS-LASER-GUI has been developed using MATLAB to model and control the critical input parameters of a $\mathrm{CO}_{2}$ laser cutting machine. Laser cutting machine possesses very complex process with respect to the influences of machining parameters, such as; cutting speed, duty cycle, frequency, power, focal distance, gas pressure, stand of distance, etc. The selection of machinability data has played an important role in the effectiveness of machine tool utilization which directly influences the overall product quality. The influences of the machining parameters on machine tools are not always precisely known and hence, it becomes difficult to recommend the optimum machinability data during machining.

ANFIS Graphical User Interface (GUI) development for laser processing: ANFIS Model using neuroadaptive learning techniques which are similar to those of neural networks was originally presented by Jang ${ }^{[6]}$. Given an input/output data sets, ANFIS constructs Fuzzy Inference System (FIS) whose membership function parameters are adjusted using backpropagation algorithm or other similar optimization techniques. Nariman-Zadeh ${ }^{[7]}$ has used hybrid genetic and SVD methods to design ANFIS networks and $\mathrm{Lo}^{[8]}$ has applied ANFIS modeling to predict surface roughness of end milled products. Yeh ${ }^{[9]}$ used ANFIS for inverse prediction of square hole profiles in boring operation. Development and customization of desired graphical user interface using MATLAB is possible and very much appreciated, where, users are no longer need to rely on programs such as $\mathrm{C}++$ or visual basic to integrate machining processes and artificial intelligent tools. The purpose of GUI is to make the work handling easier and least complicated by embedding radios buttons and pull down menus where, operators only handle graphic objects while the program automates the work process behind the screen. GUIDE (Graphic User Interface Developing Environment) is an application within MATLAB used to create the GUI. The application has the entire graphics interface which can be used to develop the interface and utilized by researchers of various disciplines.

Laser machining: Laser beams are used extensively for a variety of material-removal applications because they provide highly concentrated energy sources that can be easily transmitted and manipulated. Micromechanical structures are becoming more common with the ever increasing demand for new micro-fabrication tools. As feature sizes become smaller and smaller, i.e., typically below $100 \mu \mathrm{m}$, conventional mechanical approaches for cutting, drilling and shaping materials may be replaced with photon or particle beam techniques that enable geometric features as small as laser wavelengths (smaller than a micrometer) to be created with a high degree of precision and repeatability. In addition, laser fabrication processes are non-contact, dry and clean operations that enable ease of automation. The nonlinear behavior of the lasermaterial interactions plays a significant role in forming the final surface profile and the resultant geometry of the machined micro-features. The need to precisely control a large number of parameters, often with random components, makes the task of improving the process performance very difficult. Moreover, modeling all these factors using conventional, analytical and numerical methods poses a substantial challenge. In practice, the operator has to perform number of experiments to set the appropriate process control parameters related to the laser apparatus, motion control system and workpiece materials. This trial-anderror approach is costly and time consuming especially for a small batch production or prototyping and does not ensure near optimality with a given set of process conditions and manufacturing objectives. Laser cutting is used in precision industries as it has the ability to cut complex profiles featuring extra ordinary shapes, corners, slots and holes with small region of Heat Affected Zone (HAZ). In laser machining, surface roughness is one of the most important quality evaluation factors besides kerf width. The surface roughness is generally dependent upon the properties of the work material being cut, workpiece thickness, focal length, stand of distance, gas pressure, cutting speed, etc. including the type of cutting gas. Besides the investigation of $\mathrm{CO}_{2}$ laser cutting parameters, investigations are also being studied to further understand the relationship between the gas and the cutting parameters to obtain highly précised cut quality.

Experimental setup and procedure: In this experiment, seven input parameters were controlled, namely; stand off distance, focal distance, gas pressure, 
power, cutting speed, frequency and duty cycle. A nozzle diameter of $0.5 \mathrm{~mm}$ was used with focused beam diameter of $0.25 \mathrm{~mm}$. Material used in this experiment is grade B, Manganese-Molybdenum pressure vessel plate with a nominal gauge thickness of $5.0 \mathrm{~mm}$ and Tensile Strength of $690 \mathrm{MPa}$. The plate was cut to the dimension about $1.2 \mathrm{~m}$ length and $0.7 \mathrm{~m}$ width. A cut length of $20 \mathrm{~mm}$ performed over all the 128 profiles on the plate. Total of 128 experiments have been conducted based on the DOE matrix. All the experimental data sets and the objective functions have been incorporated into the developed ANFIS-GUI to optimize the conditions of both laser and ANFIS in developing sound predictive model. A schematic view of laser machining experimental setup is shown in Fig. 1. The machining conditions were as shown in Table 1 .

\section{Experimental parameters, machines and equipments:}

Laser machine:

- Model: Helius hybrid $2514 \mathrm{CO}_{2}$ laser cutting machine

- Controller: FANUC series 160 i-L

- Maximum capacity: 3000 watt

- Laser source that use to create laser beam is $\mathrm{CO}_{2}$ gas. The real ingredient is mixture of N2 (55), He (40) and $\mathrm{CO}_{2}$ (5) with purity $99.995 \%$

- $\quad$ Pressure $=$ Max 20 bar

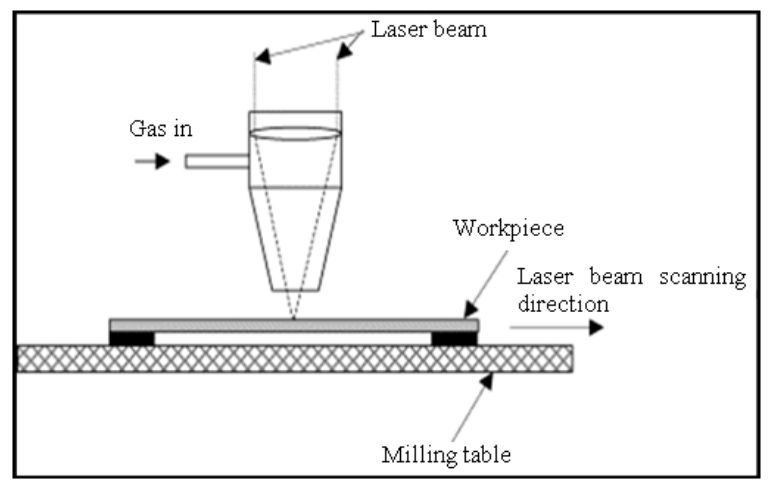

Fig. 1: Schematic of laser machining

Table 1: Machining conditions

\begin{tabular}{lrr} 
& & \\
& Level & \\
Variables & \multicolumn{1}{c}{ Low } & High \\
\hline Power (Watt) & 2500.0 & 2800.0 \\
Cutting speed $\left(\mathrm{mm} \mathrm{min}^{-1}\right)$ & 800.0 & 1200.0 \\
Frequency (Hz) & 800.0 & 1000.0 \\
SOD (mm) & 1.0 & 1.5 \\
FD (mm) & 0.0 & 1.0 \\
Pressure (Bar) & 7.0 & 10.0 \\
Duty cycle (\%) & 40.0 & 80.0 \\
\hline
\end{tabular}

\section{Surf tester:}

- $\quad$ Mitutoyo Surftest SJ301

- $\quad$ Sampling length range $(0.8 \sim 8)$

Work material:

- $\quad$ DIN 17155 HII standard

- $5.0 \mathrm{~mm}$ manganese-molybdenum

- Grade: B

- $\quad$ Tensile strength: 550-690 MPa

\section{Data collection and interpretations:}

- All the experimental materials, procedures, data collections, analysis are conducted as per the standard recommendations of 'Laser Cutting of Metallic Materials' German Standard, DIN 2310-5

- The DIN EN ISO 9013: 2000 is referred as it gives the terminological definitions and describes criteria for evaluating the quality of cutting surfaces, quality classifications and dimensional tolerances. It applies in the case of laser beam cuts from material thickness of between 0.5 and $40 \mathrm{~mm}$

\section{MATERIALS AND METHODS}

The methodology of ANFIS modeling by developed GUI is represented in the form of a flow chart as illustrated in Fig. 2. The program is started by clicking the executable file, later, click 'ANFIS Parameter' and select membership type together with its optimization method. If appropriate epoch number is unknown, set epoch number to 100 to test over-fitting, where this will provide optimized and best epoch number for the selected variable setting. Train the data by clicking 'Train' button and observe the RMSE and APE to the satisfactory level. Once done, finalize the model and keyin the machining parameters and click 'test' for numerical and graphical output together with its comparative analysis of observed and predicted result.

ANFIS-laser interface programming: An ANFIS-GUI is developed to help users utilizing Artificial Intelligence (AI) such as ANFIS (Adaptive Network-based Fuzzy Inference System) to predict responses/output. ANFIS can predict data using Sugeno FIS (Fuzzy Inference System) to relate membership and tune it using either back propagation or hybrid method. Seven input corresponding parameters namely; power, speed, pressure, focal distance, standoff distance, frequency, duty cycle with their respective two responses; surface roughness and kerf width were used for the ANFIS training. 
The ANFIS-GUI is programmed to train 2.5 and 5.0 $\mathrm{mm}$ material and predict their responses using the setting of the seven machining input variables.

The ability of ANFIS to predict the data accurately is merely depending on the settings used to train the data sets. Part of the program codes are shown in Fig. 3 with the commands subjected for RMSE, epoch number and graphical output are used to integrate the laser data sets into ANFIS platform. Therefore, the developed GUI is linked to this syntax and command which works on background to generate the output.

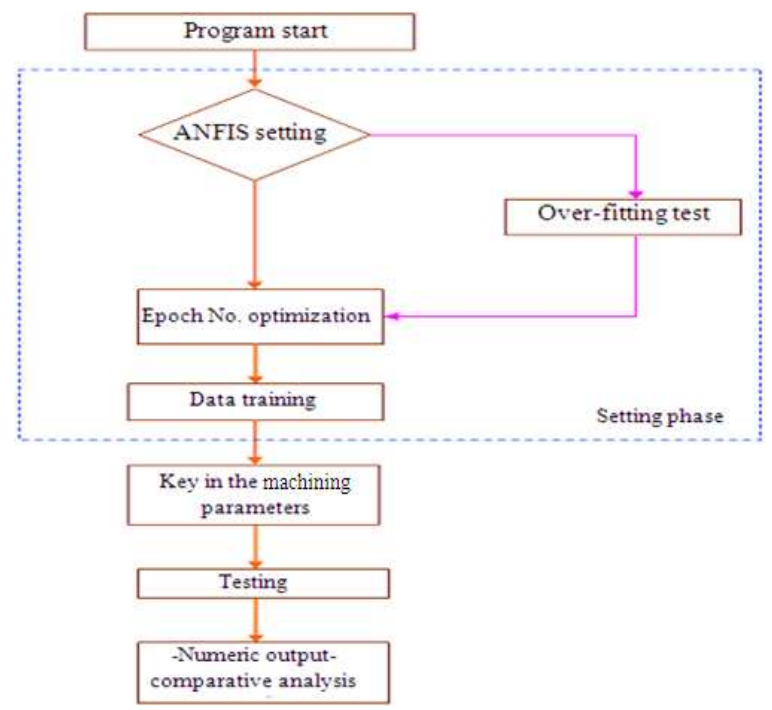

Fig. 2: GUI based ANFIS modeling methodology

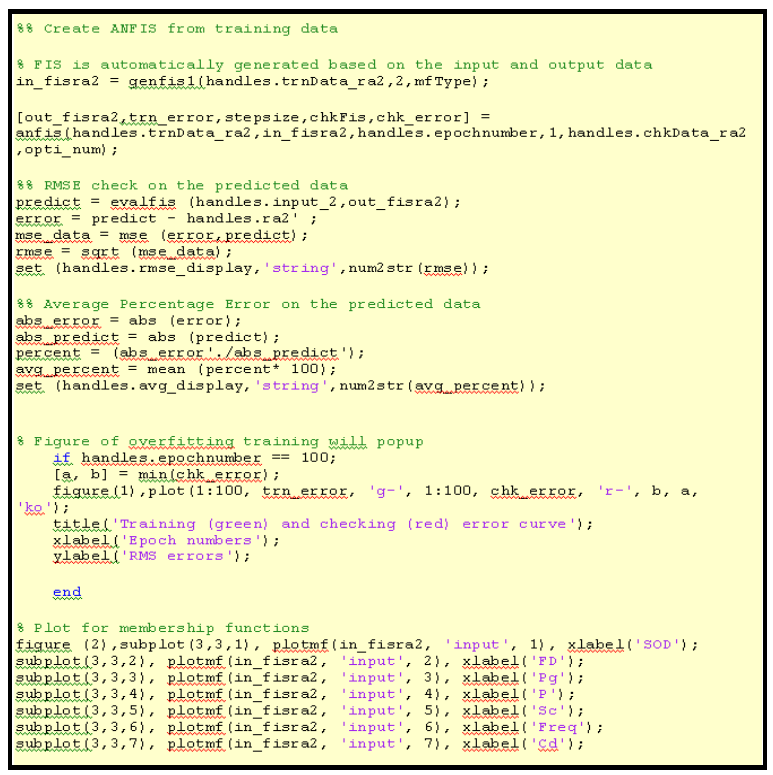

Fig. 3: Part of the programmed syntax used in integrating ANFIS- LASER-GUI

\section{RESULTS}

The developed ANFIS-LASER-GUI is the simplest user interface in modeling laser processing using ANFIS. To date, there is no such GUI developed and this can be used by almost any end user to understand the characteristics of laser processing and its significant parameters.

The GUI as shown in Fig. 4 is the completed version of ANFIS-LASER-GUI which has been created using MATLAB to predict surface roughness and kerf width for work material Mn-Mo with thickness of 2.5 and $5 \mathrm{~mm}$. The developed GUI functions to provide the fullest output of ANFIS capability in simplified steps which can be operated by almost anybody even without an engineering knowledge. The GUI can also be used to educate engineering personals to understand the fundamentals of ANFIS modeling and laser processing. Among the key features of the GUI output are; over-fitting, surface modeling, rule viewer, membership function, comparative scatter plot and numerical output. These elements are used to interpret the correlations among man, model and machine.

Over-fitting detection and optimization: The GUI is developed to detect over-fitting and help user to select the appropriate epoch number. Fig. 5 shows the dialogbox to optimize the epoch number. The optimization can be done via various setting of optimization methods and membership functions.

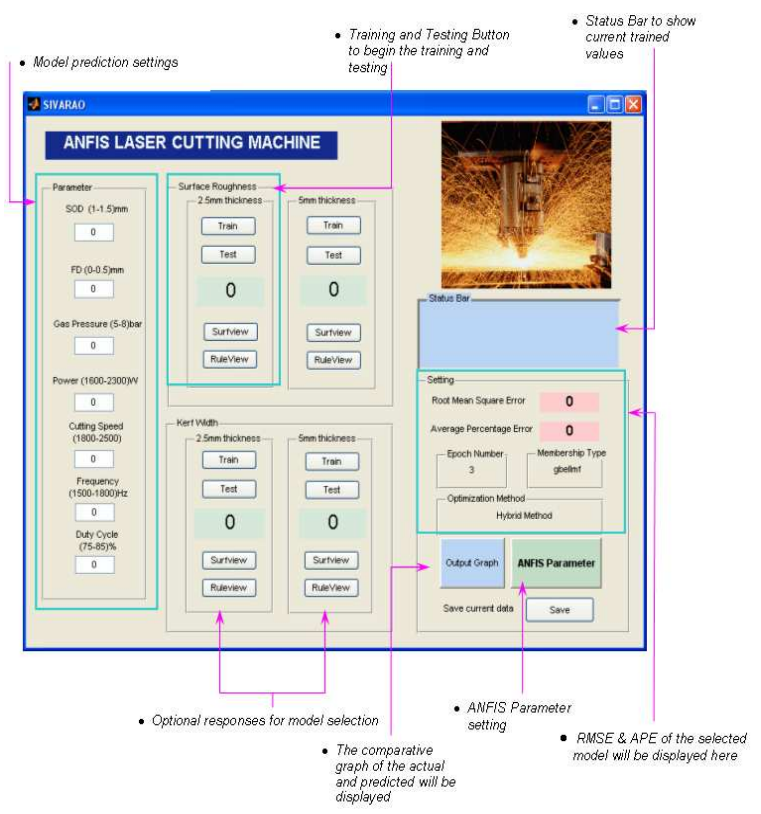

Fig. 4: Completed ANFIS-LASER-GUI for modeling of laser processing 


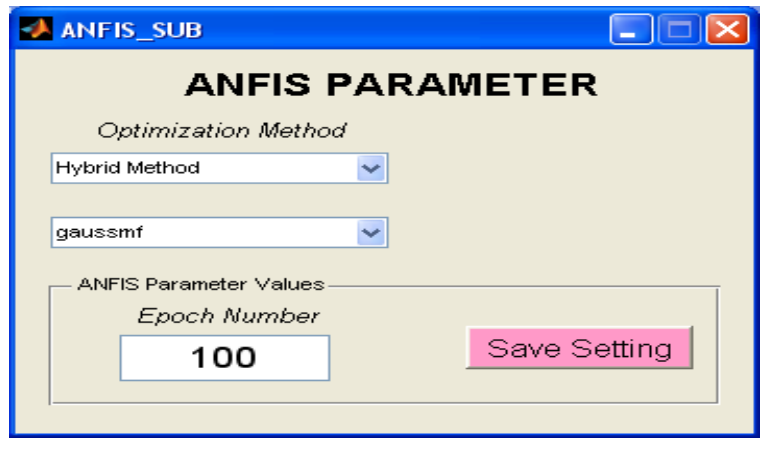

Fig. 5: Over-fitting test dialog-box

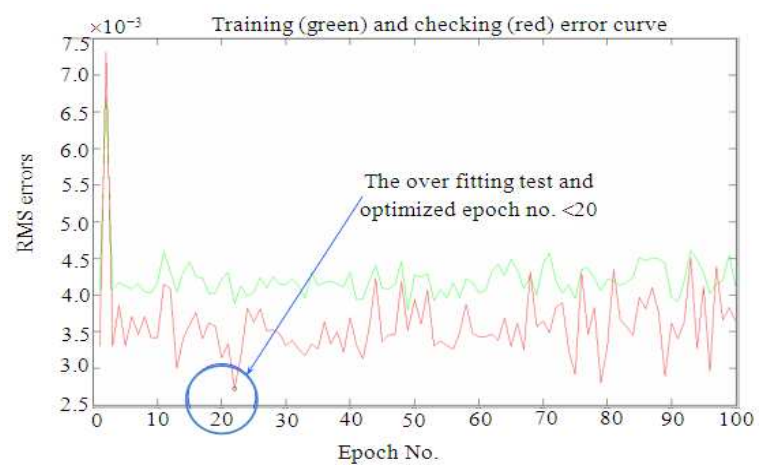

Fig. 6: Optimized epoch number prior to checking error and training error

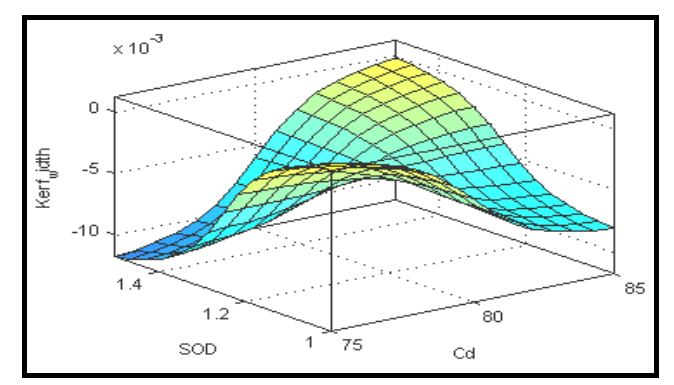

Fig. 7a: Surface model of stand of distance and duty cycle for the response of kerf width

To enable the checking of over-fitting, simply set the number of epochs to 100 and this will generate a plot displaying the checking error and training error curves and indicate optimized values. The sample of generated plot is shown in Fig. 6.

Surface model: The surface viewer is a window where user can see the correlation of the input(s) to its response via ANFIS model. The output is presented by 3D surface model as shown in Fig. 7a and b.

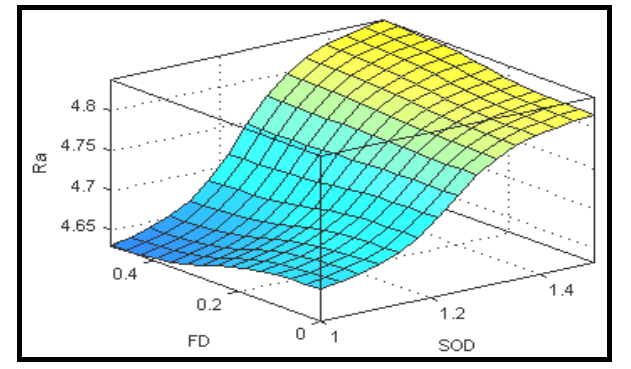

Fig. 7b: Surface model of focal distance and stand of distance for the response of surface roughness

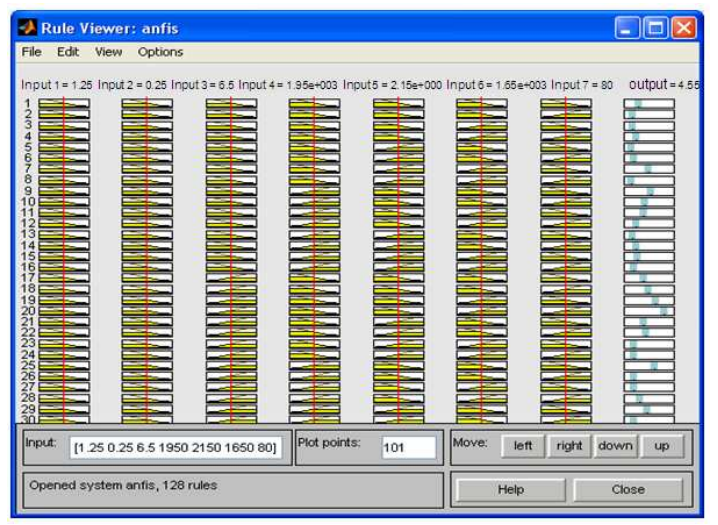

Fig. 8: The rule viewer for the ANFIS-LASER tested model

Rule viewer: Rule viewer presents a sort of micro view of the fuzzy inference system, where it displays a roadmap of the whole fuzzy inference process. It is bases the fuzzy inference diagram with a single window with plotted curve nested in it as shown in Fig. 8. The plotted curve across the top of rule viewer represents the antecedent and consequent of the first rule. Each rule is a row of plots and each column is a variable. The rule numbers are displayed on the left of each row. The first seven columns of plots show the membership functions referenced by the antecedent, or the if-part of each rule. The most right plot of the eight columns plot represents the aggregate weighted decision for the given inference system. This decision will depend on the input values for the system. The defuzzified output is displayed as a bold vertical line on this plot. The variables and their current values are displayed on top of the columns. The Rule Viewer shows one calculation at a time and in great detail.

Membership functions: The membership functions for the trained model as shown in Fig. 9 with smooth curve interaction for each parameter indicates the best fit of the developed model. 

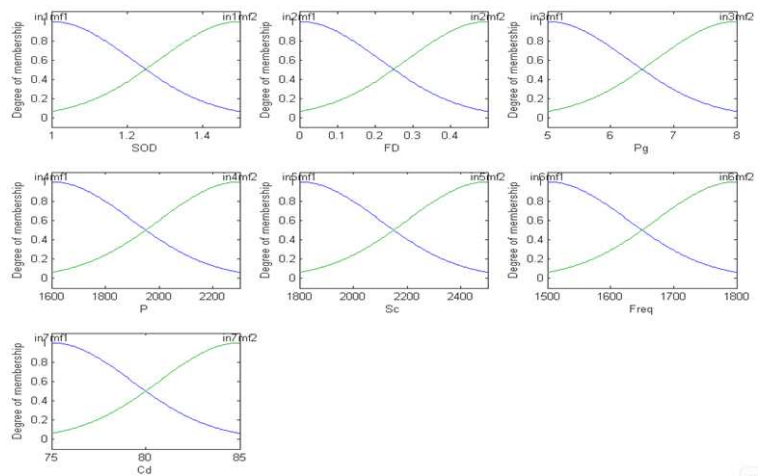

Fig. 9: Membership function of each parameter for the tested model

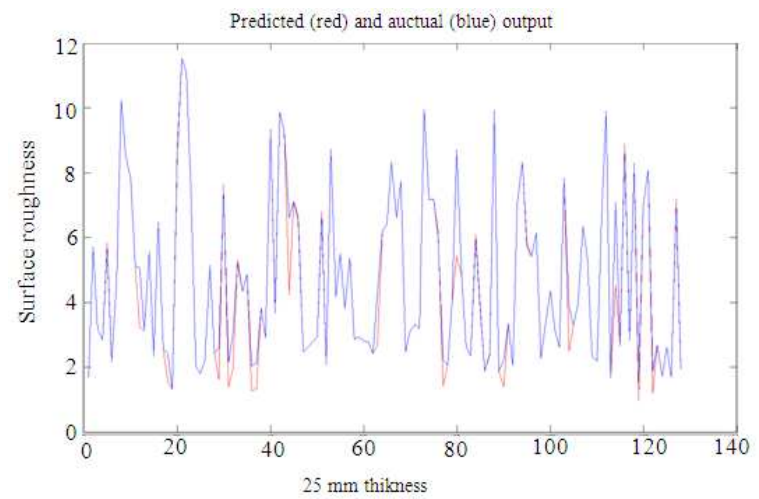

Fig. 10: ANFIS model predicted Vs observed for surface roughness

Model prediction: After the GUI is programmed and linked with the ANFIS commands via callback and MFiles, the program is then tested for its ability to predict the machining accuracy towards achieving the estimated response. ANFIS setting was selected based on comparing RMSE values between different settings. The lowest RMSE indicates the best model, perhaps it has to be observed for not being over-fit, to ensure the prediction error falls within the accepted range. The ANFIS-LASER-GUI generated output excellently correlates with the observed values performing prediction error below 9.0 percent. The agreement and comparison of both is witnessed by Fig. 10, where blue line represents the observed values while the red line represents the estimated values.

\section{DISCUSSION}

The MATLAB based Graphical User Interface (GUI) has been successfully developed and tested for its ability in modeling of laser processing by Adaptive
Network-based Fuzzy Inference System (ANFIS). It has been validated to be capable of testing the best variable setting of the intended model in ANFIS environment. This GUI enables a user even without the depth knowledge of programming and laser processing to develop a sound model by selecting significant variables on ANFIS environment. On top of that, the user can simply read the Root Mean Square Error (RMSE) and Average Percentage Error (APE) from the status bar to identify the model strength. GUI implementation on ANFIS is proved to be more efficient and ANFIS has shown the greatest prediction ability in modeling the complex and non-linear behavior of laser processing. The designed GUI is also user friendly and makes the entire steps of ANFIS modeling easier then ever. This ANFIS-LASER-GUI permits anybody for the matter to model laser machining with ANFIS for the responses of surface roughness and kerf width.

\section{CONCLUSSION}

The research work and findings conclude that, the GUI based modeling is very much practical and saves a lot of time even for a complex and non-linear process. For future studies, the comparative analysis is to be carried out between ANFIS variables and developed models to investigate extreme levels of their rapport.

\section{ACKNOWLEDGEMENT}

The researchers are obligated to sincerely thank the top level management of PT Puspetindo EngineeringManufacturing of Plant Equipments (Surabaya, Indonesia) and Kara Power Pte. Ltd. The authors would also like to thank Mr. Khor Wong Ghee, Ir. Vijayan and Ir. Segaran for their sincere guide, advice and expert knowledge sharing in making this research possible. My special thanks go to Faculty of Manufacturing Engineering, UTeM.

\section{REFERENCE}

1. Maneri, E. and W. Gawronski, 2000. LQG controller design using GUI: Application to antennas and radio-telescopes. ISA. Trans., 39: 243-264. DOI: 10.1016/S0019-0578(99)00047-6

2. Guldemir, H. and A. Sengur, 2007. Online modulation recognition of analog communication signals using neural network. Expert Syst. Appli., 33: 206-214.

http://portal.acm.org/citation.cfm?id=1223647 
3. Sokolowski, A., 2004. On some aspects of fuzzy logic application in machine monitoring and diagnostics. Eng. Appli. Artif. Intell., 17: 429-437. DOI: 10.1016/j.engappai.2004.04.016

4. Balazinski, M., 2004. Analytical modeling of tool wear progression during turning particulate reinforced metal matrix composites. CIRP. Ann. Manufactur. Technol., 54: 55-58. DOI: 10.1016/S0007-8506(07)60048-1

5. Hahn, M.L., H.L. Bing and T.L. Fu, 1995. A fuzzy neural network model for revising imperfect fuzzy rules. Fuzzy Sets Syst., 76: 25-45. http://portal.acm.org/citation.cfm?id=219430

6. Jang. R., 1996. Neuro-Fuzzy and Soft Computing: A Computational Approach to Learning and Machine Intelligence. Prentice Hall, ISBN: 0132874679, pp: 6114.
7. Nariman-Zadeh, N., 2004. Design of ANFIS networks using hybrid genetic and SVD methods for the modeling of explosive cutting process. J. Mater. Process. Technol., 155: 1415-1421. DOI: 10.1016/j.jmatprotec.2004.04.228

8. Lo, S., 2003. An adaptive-network based fuzzy inference system for prediction of workpiece surface roughness in end milling. J. Mater. Process. Technol., 142: 665-675. DOI: 10.1016/S09240136(03)00687-3

9. Yeh, F. et al., 2006. Application of ANFIS for inverse prediction of hole profile in the square hole bore-expanding process. J. Mater. Process. Technol., 173: 136-144. DOI: 10.1016/j.jmatprotec.2005.11.010 